\title{
Configuraciones, modelos de salud y enfoques basados en la Atención Primaria en Latinoamérica, siglo XXI. Una revisión narrativa*
}

\section{Configurations, health models and approaches based on Primary Health Care in Latin America, 21st century. A literature review}

\section{Configurações, modelos de saúde e enfoques baseados na Atenção Primária em América Latina, século XXI. Uma revisão narrativa}

Recibido: 29 de Noviembre de 2019. Aceptado: 10 de Febrero de 2020. Publicado: 1 de Octubre de 2020.

DOI: https://doi.org/10.11144/Javeriana.rgps19.cmse

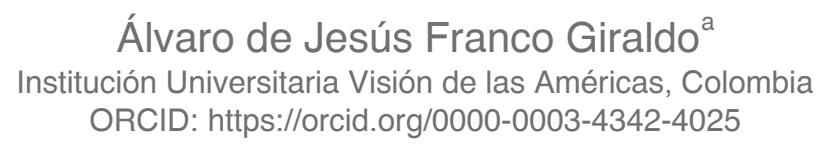

Para citar este artículo Franco-Giraldo AJ. Configuraciones, modelos de salud y enfoques basados en la Atención Primaria en Latinoamérica, siglo XXI. Una revisión narrativa. Rev Gerenc Polit Salud. 2020;19. https://doi.org/ 10.11144/Javeriana.rgps19.cmse

\footnotetext{
${ }^{a}$ Autor de correspondencia. E-mail: alvarofrancogiraldo@hotmail.com
} 


\section{Resumen}

El propósito de este artículo es aportar elementos teóricos necesarios para el desarrollo de los modelos y sistemas de salud en América Latina. Su objetivo es analizar los modelos de atención, así como las configuraciones de los sistemas de salud más relevantes, con especial énfasis en los modelos basados en la Atención Primaria en Salud (APS). Metodológicamente, se realiza una revisión narrativa (no sistemática), a partir de la búsqueda genérica en bases de datos y en google académico. Para ello, se utilizan las palabras clave: modelos, configuraciones, paradigmas y teorías/ salud. Se seleccionan aquellos artículos que den una información holística, no puntual de los modelos, y basándose en un criterio de pertinencia. Para el análisis, se establece, primero, una clasificación jerárquica de los diferentes enfoques identificados. Segundo, se analizan los modelos clásicos definidos en la literatura. Finalmente, se hace un análisis crítico de algunos modelos relacionados con la APS en América Latina y las modalidades de atención en el siglo XXI. Acerca de las relaciones estructurales necesarias para la reorientación del sistema de salud, se concluye que estas deben basarse en enfoques de APS, inclinándose hacia un "modelo de atención comunitaria" para la organización de los servicios.

Palabras clave: Sistemas de salud, tendencias, salud pública, política de salud, América Latina.

\section{Abstract}

This paper aims to provide theoretically elements to develop health models and systems in Latin America. Its objective is to analyze the attention models and the most relevant configurations of health systems, with a special focus in Primary Health Care (PHC) based models. Methodologically, a narrative no systematic review was made in databases and Google Scholar. For this purpose, it was used the following keywords: models, configurations, paradigms, and theories/health. Papers with holistic information considered relevant and appropriate were selected. For the analysis, first, a hierarchic classification of the distinct approaches identified was made. Second, classic models defined in the literature were studied. Finally, a critical analysis about some models related to PHC in Latin America and distinct modalities of attention in the 21 st century was made. With regard to the structured relationships necessary to reorient the health system, it was concluded that these must be grounded in PHC approaches, and oriented toward a "communitarian attention model" to organize services.

Keywords: Health systems, tendencies, public health, health policies, Latin America.

\section{Resumo}

O propósito deste artigo é aportar elementos teóricos necessários para o desenvolvimento dos modelos e sistemas de saúde em América Latina. Seu objetivo é analisar os modelos de atenção, bem como as configurações dos sistemas de saúde mais relevantes, com especial ênfase nos modelos baseados na Atenção Primária em Saúde (APS). Metodologicamente, realizou-se uma revisão narrativa (não sistemática), a partir da pesquisa genérica em bases de dados e em Google Acadêmico. Para isso, utilizaram-se as palavras chave: modelos, configurações, paradigmas e teorias/saúde. Selecionaram-se os artigos que deram uma informação holística, não pontual dos modelos, e baseandose em um critério de pertinência. Para a análise, se estabelece primeiro, uma classificação hierárquica dos diferentes enfoques identificados. Segundo, analisam-se os modelos clássicos definidos na literatura. Finalmente, se faz uma análise crítica de alguns modelos relacionados com a APS em América Latina e as modalidades de atenção no século XXI. Acerca das relações estruturais necessárias para a reorientação do sistema de saúde, conclui-se que estas devemse basear em enfoques de APS, se orientando por um "modelo de atenção comunitária" para a organização dos serviços.

Palavras-chave: Sistemas de saúde, tendências, saúde pública, política de saúde, América Latina. 


\section{Introducción}

En las últimas décadas, en América Latina, se han impuesto las concepciones en torno a la salud, incluidas en las propuestas de las Agencias Internacionales y Organismos Multilaterales, tras la égida neoliberal. Al tenor de ellas, se han impulsado reformas sustanciales de orden económico en los sistemas de salud con implicaciones en la práctica de la salud pública, limitando su desarrollo. Se ha olvidado, así, el devenir propio de la salud pública y de los sistemas de salud que siguen una lógica sanitaria, históricamente determinada.

Hernández-Girón et al. (1 p.316) enfatizan en que: "Un aspecto relevante de la revisión de los modelos conceptuales en salud pública se refiere a [...] la perspectiva histórica del análisis del proceso salud-enfermedad, incorporando variables epidemiológicas, ecológicas, culturales, geográficas y económicas, bajo una perspectiva interdisciplinaria [...], orientándose el análisis hacia las variables que explican comportamientos individuales, dimensiones interpersonales o grupales y las interacciones comunitarias o sociales relacionadas con la salud". A pesar de ello, en la época de la reforma neoliberal de los sistemas de salud se impusieron otras perspectivas disciplinares para la configuración de los sistemas de salud, basadas en enfoques económicos y financieros, desconociendo el componente técnico: "Si bien las reformas no tienen únicamente criterios monetarios de diseño, los mecanismos financieros constituyen parte central de su definición. Ellos toman distintas formas." (2 p.1).

Así, una de las dificultades que encontramos es la indefinición de los modelos de salud y la confusión que se da con otro tipo de modelos y configuraciones diseñados con criterios no sanitarios. De otro lado, existe una ambigüedad en los términos paradigma, modelo, configuraciones, modalidades de atención y hasta teorías y estrategias: la generalidad de los autores los denomina indistintamente, aunque responden a niveles disímiles de interpretación y para ser precisos se necesita una cierta rigurosidad académica, al diferenciar los modelos de salud de los modelos económicos y gerenciales que deberán ser complementarios.

La importancia de los modelos de salud estriba en su capacidad para disminuir la incertidumbre (3). En ese sentido, el modelo de salud es una necesidad creada desde distintas vertientes del conocimiento y de la práctica política aplicables al sector salud con el fin de estructurar unas normas y la manera de hacerla en términos de instituciones, incentivos, etc., precisar las relaciones y establecer las reglas de juego, lo que algunos plantean desde la escuela del neoinstitucionalismo $(3,4)$; con lo cual se favorece el desarrollo de la respuesta social en salud, evitando la incertidumbre; y articula las elecciones individuales y colectivas en organizaciones complejas, como la sanitarias.

En consecuencia, esta revisión tiene como objetivo explorar diferentes modelos de atención en salud y su conexidad con la práctica sanitaria, especialmente en América Latina. Asimismo, se intenta dar respuesta a esta pregunta de investigación: ¿Existen diferencias entre las configuraciones del sistema de salud, los modelos de organización de los servicios y 
las diferentes modalidades de atención? El propósito final es aportar elementos para la transformación de los sistemas de salud y de la práctica sanitaria en el siglo XXI.

\section{Método}

Los modelos buscan soluciones a problemas propios de una disciplina y de su objeto de conocimiento; en salud pública deben enfocar los problemas relacionados con la vida, la salud y la manera de enfermar y de morir de las poblaciones (5). Un modelo en salud pública debe dar cuenta de la salud de la población, y no sólo de las meras relaciones interpersonales, o de las relaciones del proceso de atención médico-paciente como se planteaba a mediados del siglo pasado (6); debe, ante todo, referirse al resultado colectivo de las intervenciones sanitarias. En su caso, el sistema de salud debe enfocarse en lo "público de la salud", que denota "un nivel de análisis $[\ldots]$ poblacional" (7).

Con esta premisa, se llevó a cabo una revisión narrativa - no sistemática- a partir de la búsqueda genérica en las bases de datos y en google académico, utilizando las palabras clave: modelos/ salud, configuraciones/salud, paradigmas/salud y teorías/salud. En un principio se consideraron los diferentes artículos que incluyeran paradigmas, sistemas, modelos de salud y modalidades de atención encontradas en las fuentes consultadas. En un segundo momento, como criterios de inclusión, se consideró la pertinencia y conveniencia de los artículos, para los objetivos de esta pesquisa. Así se seleccionaron los artículos y modelos más oportunos y adecuados. Más adelante, se establece una clasificación jerárquica de los diferentes enfoques (modelos de atención en salud); definiendo categorías del modelo de salud (macro, meso, micro), según las configuraciones del sistema de salud, el modelo de organización de los servicios y las diferentes modalidades de atención (tendencias de cambio en las organizaciones de salud). Para ello se utiliza un modelo clasificatorio basado en escalones, que representamos gráficamente como círculos concéntricos, haciéndolo coincidir con niveles de intervención en política pública, lo cual constituye un aporte de este trabajo.

Una vez seleccionados los artículos y los modelos, se analizaron los más relevantes, clásicamente definidos en la literatura científica; y se indagó por los modelos en relación con la Atención Primaria en Salud (APS), estrategia renovadora de la mayoría de los sistemas de salud en América Latina. Al final se presenta el análisis crítico de algunas modalidades de atención encontradas en la revisión de los modelos, las cuales han venido permeando las reformas de los sistemas de salud en América Latina (modelo de medicina familiar, el enfoque de riesgo, modelo de salud comunitaria, la salud familiar, modelo de gestión clínica, etc.), cuestionando su pertinencia como modelos de salud y su trascendencia para una reforma estructural. 
Configuraciones, modelos de salud y enfoques basados en la Atención

Primaria en Latinoamérica, siglo XXI. Una revisión narrativa

\section{Antecedentes sobre paradigmas, configuraciones y modelos de salud}

Si hacemos una simplificación, los modelos se pueden entender como representaciones de la realidad mediante aspectos o elementos esenciales (8-10); asimismo se concibe la teoría, como la sistematización de un determinado conocimiento, organizado de tal manera que pueda replicarse en otras circunstancias o sobre objetos similares. Las teorías buscan de representar la realidad, pero sin la solidez y el dominio de una época de pensamiento como suele suceder con los paradigmas. Así, las teorías echan mano de diversos objetos, técnicas y pronósticos de la realidad o interpretaciones de la misma; concepciones de la práctica, como formas de instrumentación e interpretación de la teoría (praxis). Esto de por sí nos hace suponer la diversidad conceptual, a partir de ideologías y relaciones diferentes que los individuos establecen con la realidad: diferencias en las praxis y en los marcos epistémicos.

Según De la Garza (11), las configuraciones en el campo de la ciencia son estructuras que no tienen por qué ser sistémicas, configuraciones que como las teóricas, o conceptuales, acepten niveles diversos de claridad y relaciones duras, de tipo causal, funcional o deductivo, o relaciones blandas (formas de razonamiento cotidiano como la analogía o la metáfora, sin dejar por fuera la contradicción, la discontinuidad o la oscuridad). Incluyen como rasgo distintivo la objetivación de relaciones sociales, artefactos, monumentos o instituciones; costumbres, rituales, mitos, reglas y códigos para dar significados, que pueden ser morales, cognitivos, estéticos, emotivos y formas de razonamiento cotidianos (basadas en contenidos culturales, relaciones de poder y económicas) (12).

Así, en salud pública, como consecuencia de los cambios globales y geopolíticos, los sistemas de salud han tomado distintos rumbos generando configuraciones variadas, dependientes de las condiciones de cada país. Al respecto, Fleury (13) plantea tres modelos de atención en salud en América Latina, en función de la reforma: modelo dual (ej., Chile), modelo universal (ej., Brasil) y modelo plural (ej., Colombia), y alude al modelo de atención cuando describe "cambios fundamentales en lo referente a su constitución política, así como en sus modalidades organizativas y financieras, y se orientan hacia una configuración más pluralista y competitiva" (13 p.34).

En el campo de las ciencias políticas, Roth (14) diferencia marco, enfoque, modelo, teoría y paradigma: un marco (o un enfoque) corresponde a la "asociación y manera de considerar los problemas, un repertorio metodológico y un vocabulario interpretativo" (14 p.70). Recordando autores clásicos de la ciencia política (Balme y Brouard, Ostrom, Sabatier), Roth aduce del enfoque o marco, que "organiza diagnósticos y prescribe interrogantes, [...] intenta identificar elementos universales [...] para clases similares de fenómenos" (14 p.70). En ese orden de ideas, un marco estructura las relaciones entre diferentes variables y las teorías se desarrollan al interior de un marco. Para Sabatier (15), desde la politología, una teoría es "una serie de proposiciones ligadas lógicamente entre ellas que buscan explicar una serie de fenómenos" (15 p.261). Roth (14), retoma a Balme y Brouard para aludir a una teoría: "un conjunto de conceptos, de variables

$\mathrm{y}$ de indicadores que establecen relaciones causales y propuestas interpretativas sobre el estado 
del mundo y sus transformaciones" (14 p.70). Y, finalmente, Sabatier define un modelo, como una "representación simplificada de un proceso (puede ser parte de una teoría) [que] precisa hipótesis en relación a un número limitado de parámetros y variables" (15 p.262).

Esquemáticamente, un modelo se inscribe en una teoría y ésta, junto con otras teorías, hará parte de un marco de análisis. El marco de análisis sirve como tal, al analista o investigador, para poder asir el objeto de estudio, bien sea una política pública o una organización, a partir de lo cual se pueden derivar las variables, las hipótesis, los indicadores, y las relaciones entre ellos, siguiendo los autores citados.

También se pueden examinar estas teorías y enfoques a la luz del concepto foucaultiano de episteme (16): "reglas generales" o "presuposiciones epistémicas inconscientes que rigen el discurso general de la cultura" en un momento histórico dado. Son, por lo tanto, formas de pensamiento relacionadas con el poder, incluyendo sus recortes ontológicos, sus puntos de vista, sus implicaciones prácticas y posicionamiento en cada época, como lo interpreta Breilh (17).

Desde otro punto de vista, varios autores $(17,18)$ han hecho aproximaciones desde la concepción de Kuhn (19) sobre paradigmas (conceptos que dominan una ciencia en determinado período de tiempo). Resaltan el papel de la estructura sanitaria, en el campo de la ciencia: los modelos y ejemplos compartidos en una comunidad científica, las creencias, valores y compromisos de esa comunidad; pero además de los razonamientos teóricos deben tenerse en cuenta otro tipo de aspectos como los modelos prácticos.

En el campo de la salud pública se han dado múltiples confusiones sobre modelos, teorías y corrientes de pensamiento, que por extensión se les ha denominado modelos. Así, se les llama inapropiadamente modelos de salud a aquellos enfoques cuyo objeto es el desarrollo del trabajo alrededor de la clínica, o al proceso de atención médica (ej. el modelo de gestión clínica), o al proceso de organización de la prestación de servicios de salud. $\mathrm{O}$, en otra dimensión, denominan modelos de salud a las configuraciones más totalizantes (ej., un sistema de salud); o a ciertas etapas del desarrollo de una disciplina, como la epidemiología, a la manera de Susser et al. (20).

En salud pública, el modelo debe interpretar la práctica política y social en consonancia con los modos de vida de una comunidad dada (la salud poblacional). Al respecto, Breilh (21) incluye en el modelo varios elementos: la dimensión ontológica (una forma de recorte del objeto salud y su interpretación), la dimensión epistemológica (formas de relación sujeto-objeto y su cosmovisión) y la dimensión praxiológica (una concepción de la práctica y posicionamiento frente a la estructura de poder). 


\section{Hallazgos}

La literatura sobre modelos de salud es cada vez más amplia: si consideramos una sola base de datos (google académico), utilizando como palabras clave "modelos de salud" encontramos 2.910 resultados, de artículos provenientes de diversas disciplinas; la búsqueda para los últimos 5 años (2015-2020) arroja 1.220 resultados; cuando se hace en los últimos 5 años pero con la expresión "modelos de salud" en el título se obtienen 247 resultados. Cuando se buscan artículos que específicamente hablen de "modelos de salud pública", los resultados son mucho menores.

La mayoría de los artículos describen experiencias de modelos de salud, en diferentes regiones y países de América Latina. Para nuestra revisión era más importante obtener artículos que abordaran el tema desde una perspectiva epistemológica, conceptual, o filosófica de la salud, que nos pudiera dar cuenta de la estructura de los modelos y de su concepción, lo que se consideró, en este caso, como un criterio de pertinencia.

Históricamente, en los artículos consultados, se reconoce el modelo biomédico, el médico social, el epidemiológico, el ecológico; mientras que en la actualidad se hace referencia más al modelo social, al familiar o al comunitario que enfatizan en una práctica sanitaria específica.

También se destaca el modelo comportamental y comunicacional (8), por ejemplo, de una de las corrientes más recientes de la promoción de la salud, que intenta cambios en los estilos de vida. Entre los modelos basados en el comportamiento y en la comunicación social, tenemos el modelo trans-teórico del comportamiento en salud, destacado por Cabrera (22), quien explica el cambio del comportamiento de acuerdo con cinco variables.

Investigadores del INSP de México (1) plantean que "la discusión sobre los paradigmas en Salud Pública origina una recapitulación histórica sobre los modelos conceptuales, desde las concepciones mágico-religiosas hasta los modelos ecológicos y socio médicos" (1 p.315). Y con Susser et al. (20), reconocen tres eras en la evolución de la epidemiología: la era de estadísticas sanitarias, con el paradigma del miasma (s. XIX); la era de la epidemiología de las enfermedades infecciosas, con el paradigma de la teoría del germen (s. XX), y la era de la epidemiología de las enfermedades crónicas (s. XXI), con el paradigma de las cajas negras. Y algunos autores afirman que emerge un nuevo paradigma por la transformación de los patrones globales de la salud y por la fuerza de las nuevas tecnologías.

También, desde la psicología se plantean varios modelos: "el modelo biopsicosocial, el modelo salutogénico, el modelo de salud positiva desde la psicología positiva, el modelo holista, el modelo de elección personal, el modelo de salud como bienestar, el modelo de salud como madurez, el modelo de salud como inteligencia emocional y el modelo de salud como resiliencia, entre otros" (23 p.70).

Más adelante, en el desarrollo de este artículo, se abordarán algunas de esas tendencias y modelos. 


\section{Los clásicos modelos y su renovación}

Siguiendo a Franco (5), encontramos el modelo médico clásico, que encarna como ninguno el modelo biologista (modelo biomédico), sin desconocer la existencia de otros más antiguos. Este modelo enfatiza en las dimensiones biológicas frente a las socioculturales, excluyendo a estas últimas de su papel en la generación de la enfermedad y, por ende, de las respuestas que pueda dar la sociedad en este campo. Paralelamente se presenta el modelo colectivo del Higienismo de los siglos XVII y XIX.

A la forma de articularse el hospital, la atención privada a los enfermos y el papel de los médicos se le ha denominado modelo médico clásico (24). Su hegemonía y orígenes se remontan al siglo XVIII y la intervención asistencial del Estado mediante el modelo biomédico era aprovechada por la clase política para legitimarse.

Al final del siglo XIX la teoría del germen coadyuva a fortalecerlo, pero las prácticas preventivas también se ven beneficiadas, lo cual favoreció el modelo preventivista epidemiológico, que irrumpe como modelo alternativo.

Como decíamos, el Higienismo con la idea sanitarista, miasmática y ambientalista se mantuvo en un segundo plano durante la segunda mitad del siglo XIX y gran parte del XX; hasta que reaparecen nuevas teorías ecologistas y ambientalistas al final del siglo XX. En ese sentido, fueron importantes las reuniones en ciudades como Oslo (1968), Río de Janeiro (1992), Sundsval (1991) y la del Protocolo de Kioto (1997), que han hecho énfasis en el tema ambiental (25); en el siglo XXI, la de los ODS - 2015 (objetivos de desarrollo sostenible) y el COP21 de París (adoptado el 15 de diciembre de 2015, dentro de la Convención Marco de las Naciones Unidas sobre el Cambio Climático) (26), alientan el espíritu de esta visión ambientalista, presionadas por la defensa urgente del planeta Tierra.

\section{Configuraciones de sistemas de salud}

Genéricamente, los modelos de salud se refieren más propiamente a los modelos de atención en salud pero, por extensión, se trasciende a los sistemas de salud, una organización más estructurada de la atención integral, la cual en este artículo denominamos configuraciones de sistemas de salud, dado su diseño en el nivel macro y su concepción de totalidad.

Los sistemas de salud se han clasificado como: sistemas segmentados, sistemas nacionales, sistemas privados, sistemas públicos, entre otros $(29,30)$. Otra clasificación fue la que utilizó el Banco Interamericano de Desarrollo (BID) en 1996, para analizar las reformas: sistemas segmentados, sistemas intensivos en el uso de contratos, sistemas públicos integrados y sistemas públicos administrados de contratos; agregándose también el modelo de competencia regulada y el concepto de pluralismo estructurado (31), por otros autores. 
En las configuraciones de los sistemas de salud se sobreponen las creencias políticas, las ideologías y las prácticas sanitarias: fluctúan desde los sistemas de salud que luchan contra la enfermedad hasta sistemas basados en los derechos (el derecho a la salud); desde sistemas públicos de servicios de salud de configuración horizontal hasta los sistemas verticales, pasando por los segmentados selectivos, fragmentados, y otros con enfoque de mercado.

Los cuatro grandes tipos de sistemas de salud originales los obtenemos atendiendo a las configuraciones históricas regionales (32): Modelo de Europa Occidental, Modelo de Estados Unidos, Modelo de Europa Oriental y Modelo del Tercer Mundo. Cada uno de los cuales tiene sus bases de conceptuales en: los esquemas de seguridad social (basados en la propuesta del canciller Alemán, Bismarck); los sistemas nacionales de salud (modelo inglés, basado en el informe Beveridge de 1942); el Modelo Semashko o Centralizado, estatista ciento por ciento (U.R.S.S.); el modelo privado (Norteamericano), el de mercado regulado, utilizando la competencia y las leyes del mercado, como mecanismo regulador; los modelos híbridos, segmentados, con mezclas de los tres primeros (América Latina); modelos públicos integrados: países Nórdicos, y modelos intermedios: con financiación pública y producción privada (Canadá, Brasil, Inglaterra post reformas).

Hoy, al renovarse el modelo de APS, las configuraciones de sistemas de salud basados en ella, también se transforman hacia los Sistemas Integrales de Salud (33).

\section{Los modelos basados en la APS}

El amanecer del siglo XXI nos despierta el afán por los Modelos basados en APS (34), buscando respuestas a diversas preguntas. No obstante, cabría anotar que sólo los modelos sociales y nacionales de carácter público han logrado hacer efectiva la APS así como las transformaciones sustantivas en la salud de la población. Lo cierto es que el mandato internacional y la gestión interna de los países han preconizado una nueva era de la APS, aún en contextos ideológicos y políticos aparentemente contradictorios con su filosofía. Cabe preguntarse ¿cuál es ese modelo?

Las estrategias y prácticas sociales propugnadas por Alma-Ata (URSS, 1978), con la APS, y Ottawa (Canadá, 1986) fundamentada en las políticas públicas, los estilos de vida, la reorganización de los servicios, la creación de ambientes saludables y la participación ciudadana, abrieron las puertas para cambiar el modelo de atención. Tres décadas después se plantea la renovación de la APS $(27,28)$.

La reorientación del modelo de salud se fundamenta en la renovación de esta estrategia. En general, se conciben varios elementos atinentes a la APS (34): un conjunto de valores (derecho al más alto nivel de salud, solidaridad y equidad); principios (responsabilidad gubernamental, sostenibilidad, intersectorialidad, participación social, etc.; y los atributos estructurantes del sistema de servicios de salud (acceso de primer contacto, integralidad, longitudinalidad, coordinación, orientación familiar y comunitaria y competencia cultural) (35). 
Al tenor de la renovación, en Chile, desde el 2005 se empezó a implantar un nuevo modelo de atención centrado en la atención primaria de salud (APS), basado en la promoción, prevención, con énfasis en la resolución ambulatoria de los problemas de salud y el uso racional y eficiente de los recursos, dando vida al Modelo de Atención Integral de Salud Familiar y Comunitaria (MAIS) (36). Chile también propone la APS basada en lo biosicosocial: "Las dimensiones consideradas por el MINSAL [...]: accesibilidad, contacto de puerta de entrada, continuidad, centrado en la comunidad, centrado en la familia, amplitud, y coordinación.” (37 p.157).

El modelo de APS es el cambio hacia un modelo de atención integral, comunitarista y con enfoque de participación social que lleva a unos fines salutogénicos de acuerdo con las más novedosas interpretaciones de la promoción de la salud (38). En Colombia, durante la etapa de la renovación, se le denominó Modelo de Atención Primaria Integral (39), que tiene como fin la satisfacción de las necesidades de salud de las personas y reordena componentes, pasando del esquema burocrático piramidal por niveles a un esquema de redes asistenciales, responsables de la atención de la salud de una comunidad.

Modelos como los descritos reconocen que la comunidad, sus redes y el primer nivel de atención, configuran la arquitectura que articula el quehacer sanitario (40). Pero los hospitales también forman parte de la continuidad de la atención contribuyendo a la resolución de los problemas de salud. Es el complemento indispensable de los sistemas integrados de servicios de salud $(33,41)$, las redes integradas, que Colombia finalmente incluye en su legislación con la Ley 1438 de 2011 y el Modelo de Atención Integral (MIAS) (41), aunque no logra reglamentarlas ni implementarlas.

En síntesis, la APS resurge hoy como un enfoque renovador en los modelos de salud, con el propósito de reorientar los servicios de salud hacia la conformación "de un conjunto de elementos estructurales y funcionales esenciales que garanticen la cobertura y el acceso universal a los servicios, de manera tal que promuevan la equidad" (34 p.IV).

\section{Otras modalidades de atención}

De la mano de la APS analizamos estas estrategias micro-organizacionales, que se podrían definir, siguiendo el modelo de la Caja Costarricense de Seguridad Social:

"Una modalidad de atención se define como una respuesta institucional organizada para atender las necesidades de salud de la población, considerando las condiciones psicosociales, culturales, biológicas y de accesibilidad a los servicios, para ofrecer al usuario(a) una atención integral con calidez y calidad basada en la evidencia" (42). Una entrevista a expertos en San José CR, 2007, afirma que las modalidades de atención contemplan diferentes aspectos del Modelo de Atención institucional, y plantea una jerarquización de categorías superior e inferior. Las superiores, los componentes mayores del Modelo de Atención de la CCSS: financiamiento, administración y 
propiedad de la infraestructura. Las inferiores, relacionadas con la organización de la atención (métodos para atender las necesidades de salud, variaciones en los programas de atención institucional, etc.).

A continuación se describen algunas de ellas:

Gestión Integral de Riesgo: estrategia para anticiparse a las enfermedades, detectar los riesgos y tratarlos precozmente, para impedir o acotar su evolución y sus consecuencias. El enfoque es individual y colectivo; clasifica las personas según su riesgo; implementa acciones de eliminación y mitigación del riesgo; traza rutas de atención para diferentes grupos de riesgo, diferenciales según riesgo (41).

Gestión Clínica: sistemas de incentivos para pacientes y agentes del sistema (pago por resultados-desempeño); "administración gerenciada" para lograr eficiencia, indemnizatoria del aseguramiento. El nivel micro enfocado a la gestión clínica presenta varias modalidades según Castaño (44): "gestión de la enfermedad", "modelos de atención de crónicos", o basados en "hogar de salud", o en "rendición de cuentas", o "unidades de práctica integrada", "las redes facilitadas", entre otras.

Los Modelos Comunitarios: después de publicada la Declaración de Astaná sobre APS (45), se refuerza la salud comunitaria:

Apoyamos la implicación de las personas, las familias, las comunidades y la sociedad civil mediante su participación en la elaboración y aplicación de políticas y planes que repercutan en la salud. (45 Declaración de Astaná art. VI).

El informe SESPAS 2018 (46) es un buen instrumento para comprender la Salud Comunitaria. En la reseña de este se lee:

El 'quien' de la salud comunitaria [...] describe los agentes principales que trabajan en la salud comunitaria -comunidad, sistema sanitario y administración local, cómo ha evolucionado su relación e implicación con la salud [...]. El ‘cómo’ recoge aportaciones metodológicas orientadas a la implementación práctica de las intervenciones comunitarias -herramientas para promover la participación, creación de redes de actividades, etc. (46 p.1).

¿Pero qué es salud comunitaria? De acuerdo con Gofin et al. (47) "la orientación comunitaria de la atención primaria está basada en los principios de la medicina comunitaria” (47 p.177), basada en las necesidades de salud de la comunidad y la respuesta a esas necesidades, en el estado de salud de la comunidad y en qué se ha hecho por parte de la comunidad. Para otros, salud comunitaria, significa que "trabajamos en un proyecto para mejorar el estado de salud de una comunidad o un colectivo" (48 p.6). 
Salud Familiar/ Medicina Familiar: carácter integral, familiar y social, fundamentado en el cuidado integral de las familias (prevención, autocuidado, gestión y promoción); con responsabilidad familiar y comunitaria, basado en estilos de vida saludables y acción multisectorial; garantiza continuidad de la atención, trabaja en red sanitaria y social $(41,43)$. Un ejemplo en América Latina es el modelo de salud familiar cubano, el cual se fundamenta en seis dimensiones: "contexto socioeconómico y cultural, composición del hogar, procesos críticos de vida familiar, relaciones intrafamiliares, afrontamiento familiar y apoyo social” (49 p.334), y basado en la atención primaria.

\section{Discusión}

Resumiendo, en esta revisión se han expuesto: de un lado, los enfoques clásicos basados en el modelo médico hegemónico, los enfoques ambientalistas y preventivistas, y todos los derivados de ellos; de otro lado, hemos revisado los modelos y modalidades de atención que se han forjado a la luz de la APS; asimismo, los modelos de sistemas de salud (bismarckiano, de seguridad social de Beveridge, segmentados, públicos, privados, de contrato público, entre otros mencionados). La consideración de ellos y sus antagonismos nos permiten encontrar un hilo conductor de análisis entre esas tensiones y las de los modelos de reforma de los sistemas de salud en Latinoamérica, en el actual contexto sociopolítico.

\section{Las tensiones de los modelos de atención en salud}

Al revisar teorías y modelos de atención en América Latina, nos tendremos que remontar al planteamiento ideológico recogido por Juan César García, pionero, en su clásico ensayo sobre corrientes de pensamiento en el campo de la salud (50): "La 'apropiación' por parte del Estado en el decenio de 1970 de las alternativas surgidas de la crítica a las instituciones médicas y el reconocimiento creciente del fracaso de estas medidas han revitalizado la discusión teórica sobre la articulación del campo de la salud en la sociedad" (50 p.389). Lucha teórica que ha incidido en el esclarecimiento de ese marco teórico y en la praxis de los modelos de salud en los años siguientes en el campo de la salud latinoamericano.

En un sentido más estricto, en salud pública, habría que adoptar concepciones epistémicas desde la praxis, que resultan diversas en una sociedad dada y hablar más detenidamente de las contradicciones entre los enfoques, teorías y modelos, algunos de ellos antagónicos entre sí, dependiendo de la perspectiva ideológica. Lo cual nos permite hablar de corrientes de pensamiento en salud, como bien lo describía Juan César García (50), (es el caso, hoy, de la medicina social, la salud colectiva y la salud pública convencional en América Latina); a lo cual le hemos agregado las configuraciones de sistemas de salud, para este análisis. Desde un principio, este artículo indagó por el carácter renovador de los métodos y las conexiones utilizadas por la nueva propuesta de APS, así como su potencial para transformar el modelo de salud, en particular en los sistemas de salud Latinoamericanos. 
Sobre la crítica al modelo médico hegemónico (MMH), enMenéndez (51) encontramos algunas interpretaciones, cuando subraya que el modelo biomédico hegemónico entra en crisis debido a la ampliación y medicalización de las áreas de atención y pérdida de eficacia, desarmonía entre los costos, el perfil epidemiológico dominante y su control; poca relación con el panorama epidemiológico determinado por la producción social y económica de la enfermedad; su enfoque curativo vs. el preventivo (51).

También, desde la perspectiva feminista también se ha criticado este modelo porque desde "la modernidad, su modelo científico el positivismo, [...], siguiendo el modelo médico hegemónico estudian a mujeres fuera de sus condiciones sociolaborales; invisibilizando subjetividades. Se discute el rol de la medicina, su función social y sus implicaciones sociopolíticas, desde el punto de vista feminista" (52).

Este análisis nos permite desentrañar una tensión entre el modelo médico hegemónico y la APS, el modelo alternativo. En la misma línea de otras tensiones, que se dan entre la medicina clínica (paradigma individual y reduccionista) y la medicina social (paradigma social - expansivo); entre usuarios y prestadores; entre el saber técnico y el saber popular; entre el enfoque de riesgo y el modelo epidemiológico social (integralidad de la práctica social y el Estado como garante de la accesibilidad universal, aportando suficiencia y pertinencia de los recursos).

En este contexto se impusieron las reformas del sector salud durante los años noventa en América Latina y el Caribe que transitó por profundos cambios estructurales y conductuales de los actores participantes, según Maceira quien anota que: "los múltiples y ambiciosos objetivos de estas reformas ya forman parte de la tradición sanitaria latinoamericana: alcanzar la equidad y la cobertura oportuna y necesaria en acciones de salud a partir de mecanismos financieros que permitan la sustentabilidad del modelo, a la vez que se dirijan a reducir, mediante intervenciones sociales, las fuertes desigualdades existentes en la distribución del ingreso" ( 2 p.1).

En consecuencia, hoy, la mayor tensión se da entre el enfoque de la eficiencia economicista (desarrollado por la reforma sectorial sanitaria basada en "canastas básicas" y cuasimercados) y el enfoque de reforma basado en la APS, en los valores de la equidad, la solidaridad, la libertad y el derecho a la salud. Contradicción que pone el asunto en el centro del debate ideológico actual que propende por cerrar la página de las reformas neoliberales en salud acaecidas por décadas en América Latina, y avanzar hacia las reformas estructurales, sociales y democráticas, cuyo eje de transformación en el sector salud, es el modelo basado en la APS y el derecho a la salud.

Con antelación, autores clásicos de la promoción de la salud $(53,54)$ habían mostrado una vía alternativa, al integrar la protección ambiental, la promoción de la salud y las acciones tanto preventivas como curativas de tipo personal, así como las políticas públicas, los estilos de vida saludables dentro de un contexto ecológico y holístico de la salud. Ellos reunieron las premisas que definen un modelo comunitario de la salud pública, que se pudieran apalancar en la promoción de la salud y en el modelo alterno basado en la APS de carácter integral. 
¡Pero hay que estar alerta! No caer en el enfoque restrictivo o selectivo de la APS o un modelo minimalista en lo social, de otras épocas (modelo que significó la definición de una canasta básica de prestaciones, focalizada en los grupos más desfavorecidos). De hecho, estudios recientes en Colombia (55) han demostrado las debilidades de la APS en un contexto de mercado, expresada en barreras para conectar las políticas nacionales y un sistema de salud basado en principios del mercado, situación que nos puede llevar de nuevo al fracaso.

Aún son grandes las dudas que desde el punto de vista epistemológico quedan de la APS, según crítica de Rovere (56), ante la APS selectiva. De otro lado, diversos actores han sostenido el discurso del derecho a la salud, sin embargo, esta invocación retórica ha sido poco efectiva para consolidar una política de salud con enfoque de derechos humanos (57).

Así y todo, la reorientación de los servicios con base en la APS es el puente estratégico para transformar los sistemas de salud, fundamentados en la promoción de la salud.

Al respecto, se han documentado varias experiencias Iberoamericanas, como veíamos antes con los ejemplos de Cuba (49), Costa Rica (42), Colombia (41), España (46) y Nicaragua (43). También Méjico (58) y Ecuador (59): en México se propone un modelo plural integral de APS que "permita la libre elección del médico o sistema de atención que el paciente o su familia decida, que cuente con financiamiento público o mixto, según el caso, y una garantía de calidad técnico certificada" (58 p.157); en Ecuador, el modelo intercultural ecuatoriano, el Ministerio de Salud Pública "legaliza y legitima a los actores de la medicina ancestral (hombres y mujeres de sabiduría), que fundamentan su arte de curar, ofrecer protección, mantener el equilibrio social y familiar, en el equilibrio de la naturaleza, en la interculturalidad..." (59 p.1170).

Son innovaciones muy importantes, pero tampoco nos podemos quedar en las experiencias locales; debemos articular con nuevas concepciones y teorías que revitalizan la APS, en el contexto de la globalidad, como lo propone OPS con la política de Salud Universal (60), encaminada a la garantía del derecho a la salud.

También es válido pensar este fenómeno en el contexto de los determinantes sociales de la salud, para cuyo abordaje se proponen varias teorías, la teoría sicosocial, la determinación social y economía política de la salud, las teorías eco-sociales, la eco-epidemiología, como se ha descrito con Nancy Krieger (61). Pero no solo estas, sino también la teorías sobre protección ambiental y su influencia en la determinación de la salud, de acuerdo con McMichael (62); o la teoría de los activos salutogénicos: "un activo en salud es cualquier factor o recurso que potencia las capacidades de personas, comunidades y poblaciones para mantener la salud y el bienestar [...] como factores de protección y promoción" (63). 


\section{Una propuesta de integración}

En la figura 1 se les da una ubicación jerarquizada a cada uno de los enfoques para entender sus potencialidades explicativas de mayor a menor alcance siguiendo algunas dimensiones de la realidad (macro, meso y micro); se les da mayor jerarquía a las configuraciones de los sistemas de salud, que representarían el concepto de modelo más amplio (macro) que podemos delimitar en salud pública; en un nivel intermedio (meso), se incluye la política de organización de servicios; y en la dimensión micro, está el corazón del modelo de atención.

Así como se reconoce que los fenómenos de la salud-enfermedad ocurren en diferentes dimensiones: "Ellos pueden ser variaciones... singulares [micro], entre individuos, o ser particulares [meso], entre grupos sociales..., o como movimientos generales, que corresponden a la sociedad en general, global [macro]" (64 p.3); así también se corresponde con las políticas $\mathrm{y}$ los modelos que establecemos para intervenir esa realidad, como se visualizan en la figural.

En la figura también ubicamos algunos modelos, estrategias o modalidades en boga actualmente, como APS, modelos de redes de servicios, de determinantes sociales; modelo de medicina familiar, de salud familiar, de salud comunitaria o propiamente de salud pública; modelos de riesgo, de gestión clínica, etc. Todos ellos tendrán un alcance distinto y, por ende, varios significados y connotaciones, según se alude en este artículo. Esto es importante porque las estrategias y políticas públicas que se puedan utilizar en uno u otro caso serán disímiles y no siempre pertinentes, algunas serán más integrales y otras basadas en el reduccionismo conceptual o en el individualismo metodológico.

En la figura 1, el modelo de atención en salud se ha colocado en el centro, en el corazón de la estructura partiendo del nivel micro pero con una capacidad de influir y condicionar el modelo de organización de los servicios de salud y las configuraciones más amplias de sistemas de salud, extendiéndose según los contextos, como se delinea en forma de estrella. Pero hay una relación de determinación desde las configuraciones macro y meso y de las políticas públicas que se gestan en estas dimensiones, hacia el modelo de salud propiamente, aunque éste a su vez contribuye al desarrollo de los sistemas de salud y las otras modalidades de atención relacionadas. 
Bien Público Salud

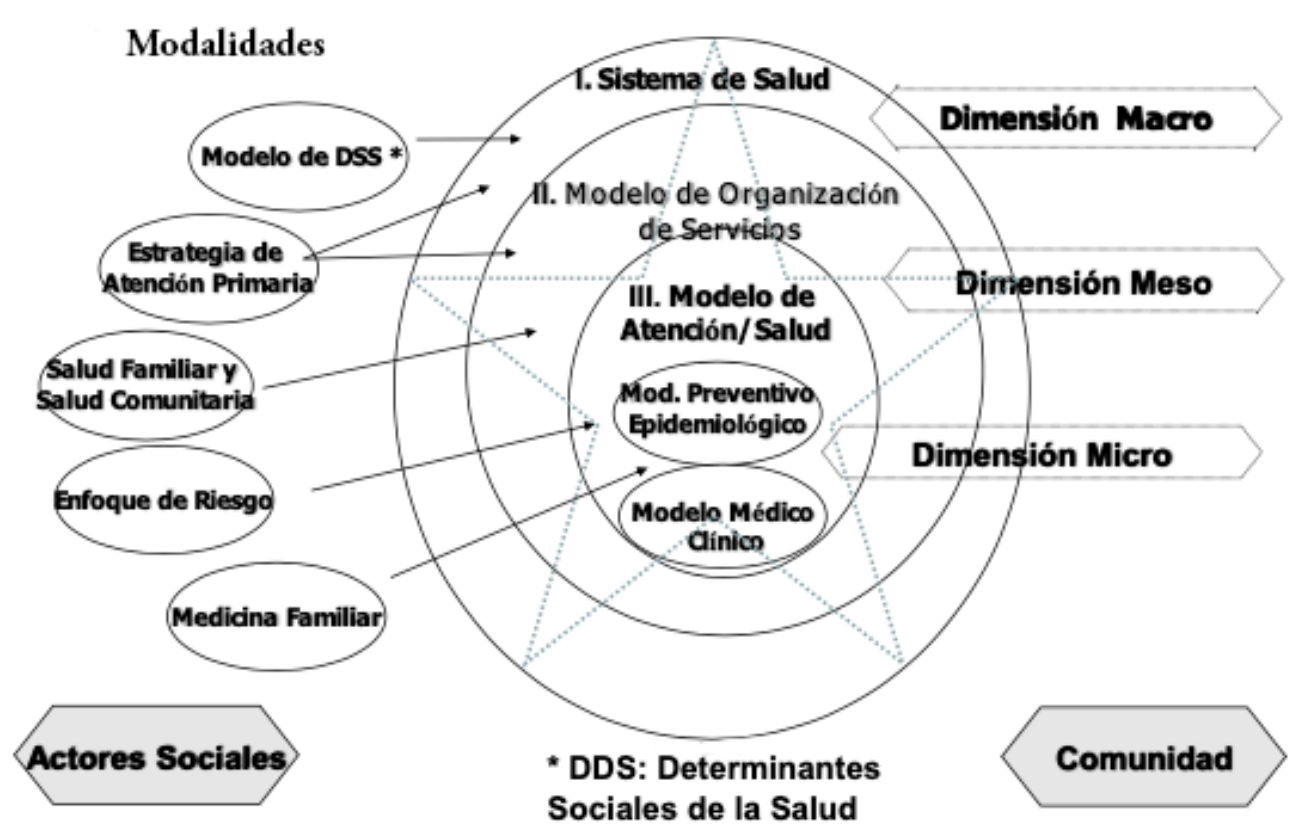

Figura 1 Modelo de salud y dimensiones de la política Fuente: elaboración propia. 
Álvaro de Jesús Franco Giraldo

\section{Conclusión}

En la literatura académica existe un abigarrado mosaico de modelos, teorías, enfoques y modalidades de atención en salud, que confunden al lector, influenciadas todas ellas por concepciones ideológicas variopintas.

Algunas de ellas giran alrededor del modelo biomédico hegemónico, alrededor del cual se han implementado propuestas modernizadoras, como aconteció con las iniciativas de reforma de los sistemas de salud en la década de 1990, de orientación neoliberal.

Ante esas políticas neoliberales basadas en el enfoque de riesgo, en el financiarismo y en la lógica curativo-preventivista de la enfermedad (modelos de gestión clínica y de atención gerenciada), los sistemas de salud en América Latina se deben reestructurar atendiendo a otra lógica, basada en las necesidades de la población y en garantizar la equidad en salud y los derechos humanos.

En consecuencia, se necesitan otro tipo de modelos en los sistemas de salud para el siglo XXI, basados en la APS integral, como eje, y siguiendo los lineamientos de la salud comunitaria, con enfoques salutogénicos de promoción de la salud y sistemas universales de salud.

\section{Referencias}

1. Hernández-Girón C, Orozco-Núñez E, Arredondo-López A. Modelos conceptuales y paradigmas en salud pública. Rev. Salud Pública. 2012;14(2): 315-324. https://www.scielosp.org/pdf/rsap/2012.v14n2/3 $15-324 /$ es

2. Maceira D. Economía Política de las Reformas de Salud: Un Modelo para Evaluar Actores y Estrategias. Nota Técnica de Salud No. 2. Washington DC: BID. Marzo; 2007. https://publications.iadb.org/publications/spanish/document/Econom\%C3\%ADa-pol\%C3\%A Dtica-de-las-reformas-de-salud-Un-modelo-para-evaluar-actores-y-estrategias.pdf

3. Grosso G. Modelo de salud. En: Grosso G, Gómez A, Betancourt VF. Modelos de salud: administración de planes de beneficios desde la perspectiva de la gestión del riesgo. Bogotá: Universidad Nacional de Colombia, 2006. http://www.bdigital.unal.edu.co/637/2/9587016084.01.pdf

4. Kalmanovitz S. La Criometría y la Historia Económica Institucional: Reflejos Latinoamericanos. Bogotá: Banco de la República; 2004.

5. Franco A. Tendencias y teorías en salud pública. Revista Facultad Nacional de Salud Pública. 2006;24(2): 119-131. http://www.scielo.org.co/scielo.php?script=sci_arttext\&pid=S0120-386X2006000200012

6. Illich I. Medical Nemesis. The expropriation of health. New York: Pantheon books./ London: Health Education Council. 1976. http://www.columbia.edu/itc/hs/pubhealth/rosner/g8965/client_edit/readi ngs/week_2/illich.pdf 
7. Frenk J. La salud de la población. Hacia una nueva salud pública. México DF: Fondo de Cultura Económica; 1994. http://bibliotecadigital.ilce.edu.mx/sites/ciencia/volumen3/ciencia3/133/htm/pobl acion.htm

8. Cabrera G. Teorías y modelos de la salud pública en el siglo XX. Colombia Médica. 2004;35(3): 164-168. http://www.redalyc.org/articulo.oa?id=28300308

9. Hernández LJ, Ocampo J, Ríos DS, Calderón C. El modelo de la OMS como orientador en la salud pública a partir de los determinantes sociales. Rev Salud Pública. 2017;19(3): 393-395. http://dx.doi.org/10 $.15446 /$ rsap.v19n3.68470

10. Vanryn M. Heany C. What's the use of theory? Health Educ Quant. 1992;19: 315-330. https://doi.org /10.1177/109019819201900304

11. De la Garza, E. La epistemología crítica y el concepto de configuración. Revista Mexicana de Sociología. 2001;63(1). http://docencia.izt.uam.mx/egt.

12. De la Garza E, Leyva G. (eds.) Tratado de metodología de las ciencias sociales: perspectivas actuales. Diciembre de 2010. https://www.academia.edu/9116048/Tratado_de_Metodolog\%C3\%ADa_de_las _Ciencias_Sociales_perspectivas_actuales_Enrique_de_la_Garza_Toledo_y_Gustavo_Leyva_

13. Fleury S. ¿Universal, dual o plural? Modelos y dilemas de atención de la salud en América Latina. Washington DC: BID - INDES. 2002. https://www.researchgate.net/profile/Sonia_Fleury/publication/239597859_UNIVERSAL_D UAL_O_PLURAL_MODELOS_Y_DILEMAS_DE_ATENCION_DE_LA_SALUD_EN_AMERI CA_LATINA/links/0deec53205e-0eb4baa000000.pdf

14. Roth AN. Perspectivas teóricas para el análisis de las políticas públicas: ¿de la razón científica al arte retórico? Estudios Políticos (Universidad de Antioquia), 2008 (julio - diciembre); 33: 67-91. https://www.researchgate.net/publication/277746346_Perspectivas_teoricas_para_el_analisi s_de_las_politicas_publicas_de_la_razon_cientifica_al_arte_retorico

15. Sabatier P (ed.). Theories of the policy process. Boulder: Westview Press/University of California, Davis. 1999. http://ruby.fgcu.edu/courses/twimberley/EVR2861/theorypolprocess.pdf

16. Foucault M. Las palabras y las cosas. México DF: Siglo XXI Editores; 1978.

17. Breilh J. Nuevos paradigmas en salud pública. En: Programa de Naciones Unidas para el Ambiente/ OPS/ Reunión internacional. "Un enfoque de ecosistemas para la salud humana: enfermedades trasmisibles y emergentes". Río de Janeiro: Escuela Nacional de Salud Pública Fiocruz. Noviembre de 1999.

18. Susser M. Glossary: causality in Public Health Science. JECH. 2001;55: 376-378. https://epiville.ccnm tl.columbia.edu/assets/pdfs/Susser2001GlossaryCausality.pdf

19. Kuhn TS. The structure of scientific revolution. Chicago: Chicago University Press; 1970.

20. Susser M, Susser E. Choosing a future for epidemiology: eras and paradigms. Am J Public Health. 1996;86: 668-673. https://www.ncbi.nlm.nih.gov/pmc/articles/PMC1380474/pdf/amjph00516-0062 .pdf

21. Breilh J. Nuevos paradigmas en salud pública. Universidad Andina Simón Bolívar (Repositorio digital), 1999. https://pdfs.semanticscholar.org/1b1d/b9eefeaf1cab12e20d8f3c9c85f4426d430c.pdf 
22. Cabrera G. El modelo transteórico del comportamiento en salud. Rev Fac Nac Salud Pública. 2000;18(2): 129-138. https://www.redalyc.org/pdf/120/12018210.pdf

23. Juárez F. El concepto de salud: Una explicación sobre su unicidad, multiplicidad y los modelos de salud. Intern J Psychol Res. 2011;4(1): 70-79. https://www.redalyc.org/pdf/2990/299022819009.pdf

24. Comelles J. La utopía de la atención integral de salud. Auto atención, práctica médica y asistencia primaria. Revisiones en salud pública. 1993;3: 171-179. https://www.researchgate.net/publication/232242196_La_utopia_de_la_atencion_integral _en_salud_Autoatencion_practica_medica_y_asistencia_primaria/citation/download

25. OPS/OMS. La Salud en las Américas. Organización panamericana de la salud. Publicación Científica 569. Vol. I. Washington DC: OPS, 1998.

26. UN. Conference of the Parties Twenty-first session Paris, 30 November to 11 December 2015. Adoption of the Paris Agreement. https://unfccc.int/resource/docs/2015/cop21/eng/109r01.pdf

27. Franco-Giraldo A. ¿APS: de regreso al pasado? Revista Facultad Nacional de Salud Pública. 2012;30(1): 83-94. http://www.scielo.org.co/pdf/rfnsp/v30n1/v30n1a10.pdf

28. OMS. Informe sobre la salud en el mundo 2008: La atención primaria de salud más necesaria que nunca. Ginebra: La Organización. 2008. https://www.who.int/whr/2008/08_report_es.pdf?ua=1

29. Medici A. Las reformas de salud en América Latina y el Caribe. En: Sánchez H, Zuleta G. La hora de los usuarios. Reflexiones sobre economía política de las reformas de salud. Washington DC: BID/ Centro de estudios Salud y Futuro; 2000.

30. OPS. La salud y la salud pública en la historia. En: OPS/OMS. Salud Pública en las Américas (Capítulo 4). Washington DC: OPS; 2000.

31. Londoño JL, Frenk J. Pluralismo estructurado: Hacia un modelo innovador para la reforma de los sistemas de salud en América Latina. BID, Research Department, RES Working Papers. Enero 1 de 1997. https://www.researchgate.net/publication/23531504_Pluralismo_estructurado_Hacia_un _modelo_innovador_para_la_reforma_de_los_sistemas_de_salud_en_America_Latina

32. Campos M, Sáez JM, Sánchez A. Análisis comparados de sistemas sanitarios. En: Martínez F, Navarro V, et al. Salud Pública. México: McGraw-Hill-Interamericana; 1998.

33. OMS/OPS. Sistemas integrados de servicios de salud. Conceptos, opciones de política y hoja de ruta para su implementación en las Américas. Washington DC: OMS/OPS, 2008.

34. OPS (Organización Panamericana de la Salud), Renovación de la Atención Primaria de Salud en las Américas: Documento de posición de la Organización. Washington DC: OPS/OMS, 2007. https://w ww.paho.org/hq/dmdocuments/2010/Renovacion_Atencion_Primaria_Salud_Americas-OPS.pdf

35. Harzheim E, Casado V, Bonal P. Eurosocial/ Europeaid. Grupo de Pesquisa em Atenção Primária à Saúde de Porto Alegre, GP. APS. Harzheim E. (coord.) Documento Marco. La formación de profesionales de salud para la APS y Salud Familiar y Comunitaria en América Latina. Porto Alegre, septiembre de 2009. http://www.msal.gov.ar/observatorio/images/stories/documentos_formacion/ac erca_de/HARZHEIM.CASADOVICENTE-Formacion_profesional_para_la_APS.pdf 


\section{Configuraciones, modelos de salud y enfoques basados en la Atención}

Primaria en Latinoamérica, siglo XXI. Una revisión narrativa

36. García-Huidobro D, Barros X, Quiroz A, Barría M, Soto G, Vargas I. Modelo de atención integral en salud familiar y comunitaria en la atención primaria chilena. Rev Panam Salud Publica. 2018;42: 160. https://doi.org/10.26633/RPSP.2018.160

37. Moraga-Aranda C, Leiva-Pereira J. Modelo de atención de salud familiar: representaciones sociales de los funcionarios de atención primaria. Salud \& Sociedad. 2013;48(2): 156-167. https://doi.org/10.2 2199/S07187475.2013.0002.00004

38. Antonovsky A. The salutogenic model as a theory to guide health promotion. Health Promot Int. 1996;11(1): 11-18. https://academic.oup.com/heapro/article/11/1/11/582748

39. Vega R, Acosta N, Mosquera PA, Restrepo, O. Atención Primaria Integral. Bogotá: Alcaldía Mayor de Bogotá / PUJ; 2009.

40. Gobierno de Chile. Subsecretaria de redes asistenciales/ División de atención primaria: Orientaciones para la planificación y programación en red, año 2013. Septiembre de 2013. http://web.minsal.cl/po $\mathrm{rtal} / \mathrm{url} /$ item/cd34d7da59bfbd48e0400101650154ea.pdf

41. MSPS (Ministerio de Salud y Protección Social). Comité Ampliado. Lineamientos generales para el desarrollo del modelo integral en atención salud para el sistema general de seguridad social en salud - SGSSS. Bogotá. Julio de 2014.

42. Caja Costarricense de Seguro Social (área de atención integral a las personas). Lineamientos metodológicos para el diseño de propuestas de modalidades de atención en los establecimientos de salud. Metodología para la elaboración de modalidades de atención. San José de CR, 2008. https:// www.binasss.sa.cr/libros/lineamientos09.pdf

43. Nicaragua. Ministerio de Salud. Gobierno de Reconciliación y Unidad Nacional. Marco Conceptual del Modelo de Salud Familiar y Comunitario (MOSAFC) MINSA, 2 ${ }^{\mathrm{a}}$ ed. Managua, marzo 2007. https://www.paho.org/nic/index.php?option=com_docman\&view=download\&category_slug =publicaciones-anteriores \&alias $=230$-modelo-de-salud-familiar-y-comunitario $\&$ Itemid $=235$

44. Castaño RA. Mecanismos de pago en salud: anatomía, fisiología y fisiopatología. Bogotá: ECOE Ediciones; 2014.

45. OMS. Conferencia Mundial sobre Atención Primaria de Salud. Desde Alma-Ata hacia la cobertura sanitaria universal y los Objetivos de Desarrollo Sostenible. Astaná (Kazajstán), 25 y 26 de octubre de 2018. https://www.who.int/docs/default-source/primary-health/declaration/gcphc-declaration-sp.pdf

46. García MH, March S, Botello B, Cubillo J, Gea V, Segura A (Editores del Informe SESPAS 2018). Salud comunitaria y administración local (Nota editorial). Gaceta Sanitaria. 2018;32(S1): 1-4. https://ww w.gacetasanitaria.org/es-pdf-S0213911118301869

47. Gofin J, Gofin R. Atención primaria orientada a la comunidad: un modelo de salud pública en la atención primaria. Rev Panam Salud Publica/Pan Am J Public Health. 2007;21(2/3): 200. https:/www.scielo sp.org/article/rpsp/2007.v21n2-3/177-185/es/

48. Armas CS, Hernán M, Cofiño R. ¿De qué hablamos cuando hablamos de «salud comunitaria»? (Informe SESPAS 2018: Salud comunitaria y administración local). Gaceta Sanitaria octubre. 2018;32(S1): 5-12. https://www.gacetasanitaria.org/es-pdf-S0213911118301547 
49. Louro Bernal, I. Modelo de salud del grupo familiar. Rev Cubana Salud Pública 2005;31(4): 332-337. http://scielo.sld.cu/scielo.php?script=sci_arttext\&pid=S0864-34662005000400011

50. García JC. Medicina y sociedad-Las corrientes de pensamiento en el campo de la salud. Educ Med Salud. 1983;17(4): 363-397. https://www.google.com/search?q=Educ+M\%C3\%A9d+Salud\%2C+Vol.+17\%2C+No. +4 $+(1983) \& r l z=1 C 1 P R F C$ enCO730CO731\&oq=Educ $+\mathrm{M} \% \mathrm{C} 3 \% \mathrm{~A} 9 \mathrm{~d}+\mathrm{Salud} \% 2 \mathrm{C}+\mathrm{Vol} .+17 \% 2 \mathrm{C}+\mathrm{No}$ $.+4+(1983) \&$ aqs $=$ chrome..69i57.1777j0j8\&sourceid $=$ chrome\&ie $=U T F-8$

51. Menéndez E. Modelo Médico Hegemónico y Atención Primaria. En: Memorias Segundas Jornadas de Atención Primaria de la Salud. 30 de abril al 7 de mayo. Buenos Aires; 1988.

52. Antequera MC. Análisis desde el punto de vista feminista del rol de la medicina en el modelo de salud médico-hegemónico. VII Jornadas de Jóvenes Investigadores. Instituto de Investigaciones Gino Germani, Facultad de Ciencias Sociales, Universidad de Buenos Aires; 2013.

53. Millio N. Ciudades sanas. La nueva salud pública y la investigación que necesitamos. Revisiones en Salud Pública. 1993;3: 155-167.

54. OMS/ Asociación Canadiense de Salud Pública. Carta de Ottawa para la promoción de la salud. Ottawa (Canadá). 1986. https://www.paho.org/hq/dmdocuments/2013/Carta-de-ottawa-para-la-apromocionde-la-salud-1986-SP.pdf

55. Mosquera PA, Hernández J, Vega R, Labonte R, Sanders D, Dahlblom K, San Sebastián M. Challenges of implementing a primary health care strategy in a context of a market-oriented health care system: The experience of Bogotá, Colombia. Int J Health Plann Mgmt, 2014;29(4): 347-367. http://onlinel ibrary.wiley.com/doi/10.1002/hpm.2228/full

56. Rovere, M. Atención Primaria de la Salud en Debate. Saúde Em debate. Revista do centro brasileiro de estudos de saúde (Rio de Janeiro). 2012;36(94): 327-343. https://www.scielo.br/scielo.php?script=s ci_arttext\&pid=S0103-11042012000300003\&lng=en\&nrm=iso\&tlng=es

57. Ase I, Burijovich J. La estrategia de Atención Primaria de la Salud: ¿progresividad o regresividad en el derecho a la salud? Salud Colectiva (Buenos Aires). 2009;5(1): 27-47. http://revistas.unla.edu.ar /saludcolectiva/article/view/229

58. Jaime JJ, Vargas-Hernández JG. Modelo “plural-integral” de Atención Primaria a la Salud y satisfacción de los usuarios. Criterio Libre (Bogotá). 2013;11(18): 155-158. https://revistas.unilibre.edu.co/inde x.php/criteriolibre/article/view/1129/867

59. Almeida L, Almeida L. Fundamentación del modelo de gestión intercultural ecuatoriana en la atención primaria de salud. MEDISAN. 2014;18(8): 1170-1183. http://scielo.sld.cu/scielo.php?script=sci_abs tract\&pid=S1029-30192014000800019\&lng=es\&nrm=iso\&tlng=es

60. Organización Panamericana de la Salud (OPS). Salud Universal en el Siglo XXI: 40 años de Alma-Ata. Informe de Comisión de Alto Nivel. Washington DC: OPS. 2019. http://iris.paho.org/xmlui/bitstrea $\mathrm{m} /$ handle $/ 123456789 / 50960 / 9789275320778$ spa.pdf? sequence $=5 \&$ isAllowed $=\mathrm{y}$

61. Krieger N. Theories for social epidemiology in the 21 st century: an ecosocial perspective. Int $J$ Epidemiol. 2001;30(4): 668-677. https://academic.oup.com/ije/article/30/4/668/705885 
62. McMichael AJ. Environmental and social influences on emerging infectious diseases: past, present and future. Philos Trans R Soc Lond B Biol Sci. 2004;359(1447): 1049-1058. https://www.ncbi.nlm.nih .gov/pmc/articles/PMC1693387/m8c0nsultado

63. Fernández RD. Comunidades saludables; una propuesta de Acción Comunitaria para la Promoción de Salud desde el Modelo de Activos en el Barrio de la Barceloneta. Memoria Proyecto Final de Master. Universidad Autónoma de Barcelona: Master Oficial en Participación y Políticas Sociales, UAB; 2013.

64. Castellanos PL. Sobre el concepto de salud-enfermedad. Descripción y explicación de la situación de salud. Boletín Epidemiológico OPS. 1990;10(4): 1-7. https://iris.paho.org/bitstream/handle/10665.2 /32628/8366.pdf?sequence=1\&isAllowed=y

Notas

* Artículo de revisión. 